\title{
miR-205-5p downregulation decreases gemcitabine sensitivity of breast cancer cells via ERp29 upregulation
}

\author{
CHANGPO MA, XUEJUN SHI, WENCHAO GUO, FUKAI FENG and GUANGSHUN WANG
}

Thoracic Surgery Department, Tianjin Baodi People's Hospital, Tianjin 301800, P.R. China

Received August 18, 2018; Accepted June 27, 2019

DOI: $10.3892 /$ etm.2019.7962

\begin{abstract}
Breast cancer is the most common cancer in women worldwide, and the incidence and mortality rates are increasing every year. Dysregulation of microRNAs (miRNAs or miRs) is an important step in the initiation and development of breast cancer. Previous studies demonstrated that miR-205-5p is closely associated with occurrence and development of breast cancer; however, underlying mechanisms remain unclear. In the present study, reverse transcription-quantitative polymerase chain reaction assays were used to analyze miR-195-5p and endoplasmic reticulum protein 29 (ERp29) levels in breast cancer and matched normal tissues. Western blot analysis was performed to analyze ERp29 and heat shock protein 27 (HSP27) protein expression levels. Cell viability, flow cytometry and luciferase reporter assay were used to examine cell proliferation, apoptosis and direct miRNA-mRNA binding, respectively. The results revealed that miR-205-5p expression in breast cancer tissues and cell lines was decreased compared with normal tissues and a normal cell line. Overexpression of miR-205-5p significantly augmented cytotoxicity effects of gemcitabine treatment in MDA-MB-231 and BT549 cells. It was observed that miR-205-5p negatively regulated ERp29 expression in breast cancer cells. Dual luciferase assays confirmed that ERp29 was a target of miR-205-5p in breast cancer cells. Additionally, following the established gemcitabine-resistant MDA-MB-231 cells (MDA-MB-231/GEM), ERp29 and HSP27 expression was upregulated and miR-205-5p was downregulated compared with parental cells. Overexpression of miR-205-5p reversed gemcitabine resistance in MDA-MB-231/GEM cells. In conclusion, the present study indicated that miR-205-5p may inhibit gemcitabine resistance in breast cancer cells via inhibition of ERp29 expression.
\end{abstract}

Correspondence to: Dr Guangshun Wang, Thoracic Surgery Department, Tianjin Baodi People's Hospital, 8 Guangchuan Road, Tianjin 301800, P.R. China

E-mail: wangguangshun207@sohu.com

Key words: breast cancer, microRNA-205-5p, endoplasmic reticulum protein 29

\section{Introduction}

Breast cancer exhibits the highest incidence and mortality rates among women worldwide. According to a statistical analysis of cancer cases in 2012 (1), the number of new breast cancer cases and cases of mortality are $\sim 1,700,000$ and 521,900, respectively. Breast cancer accounts for $25 \%$ of all cancer cases and $15 \%$ of cancer-associated cases of mortality (2).

Gemcitabine (2',2'-difluoro-deoxycytidine; $\mathrm{dFdC})$ is a nucleoside analog that prevents DNA replication and transcription leading to apoptosis (3). Preliminary clinical observation indicated that the effectiveness of gemcitabine in the treatment of advanced breast cancer was 14-27\% (4,5). Advanced breast cancer chemotherapeutic drugs recommended by the Chinese breast cancer diagnosis and treatment guide (2017) include gemcitabine (6). Gemcitabine has good therapeutic effects in a variety of patients with cancer, including the elderly (7). However, gemcitabine resistance is common, and the underlying molecular mechanisms remain to be elucidated (8).

The endoplasmic reticulum (ER) protein 29 (ERp29) is a member of the ER chaperones that does not contain an active-site double-cysteine motif with oxidoreductase activity, observed in other ER chaperones (9). The structural variation of ERp29 suggests a varying function in cells, particularly cancer cells (9). Recent studies demonstrated that ERp29 overexpression resulted in G0/G1 arrest in MDA-MB-231 cells, leading to a marked delay in tumorigenesis onset in vivo (10). ERp29 upregulated heat shock protein 27 (HSP27) expression in cancer cells $(11,12)$ and attenuated apoptosis caused by chemotherapy drugs (13). Another study revealed that ERp29 served a key role in the development of gemcitabine resistance in cancer cells (14).

microRNAs (miRNAs or miRs) are small non-coding RNA species containing 22-25 ribonucleic acids, which are major elements of gene expression control, causing translation inhibition or mRNA degradation $(15,16)$. It is widely reported that miRNAs serve important roles in tumorigenesis $(17,18)$. miR-205-5p is a highly conserved miRNA that is expressed in mammary gland progenitor cells and in stratified squamous epithelium-derived tissues $(19,20)$. A previous study suggested that miR-205-5p is downregulated in breast cancer $(21,22)$. As one of the most researched miRNAs in breast cancer, it is involved in cellular differentiation, migration and proliferation $(18,19)$. However, the role of miR-205-5p in the regulation of gemcitabine sensitivity has not been elucidated in breast cancer. 
In this study, miR-205-5p expression was downregulated and cell viability was decreased in a gemcitabine-resistant breast cancer cell line (MDA-MB-231/GEM) compared with the parental cell line (MDA-MB-231/P). miR-205-5p bound to the $3^{\prime}$ untranslated region (3'-UTR) of ERp29 directly and negatively regulated its expression. Taken together, this study revealed that miR-205-5p may mediate gemcitabine resistance in breast cancer by targeting ERp29.

\section{Materials and methods}

Clinical tissue samples. A total of 25 breast cancer and matched adjacent non-tumor tissue samples were collected at the Tianjin Baodi People's Hospital between July 2014 and February 2017 (Tianjin, China). All tumor tissue samples were obtained from women aged $30-65$ years (mean age, 46 years). Tissue samples were immediately frozen in liquid nitrogen after surgery and stored at $-80^{\circ} \mathrm{C}$ until use. The present study was approved by the Ethics Committee of Tianjin Baodi People's Hospital. Written informed consent was provided by all participants.

Cell culture and reagents. MDA-MB-231 and BT549 human breast cancer cell lines were purchased from the American Type Culture Collection (ATCC). MDA-MB-231 and BT549 cells were cultured in Dulbecco's modified Eagle's medium (DMEM; Thermo Fisher Scientific, Inc.) supplemented with $10 \%$ fetal bovine serum (HyClone; GE Healthcare Life Sciences) and $1 \%$ penicillin-streptomycin solution (Thermo Fisher Scientific, Inc.) in a humidified atmosphere containing $5 \% \mathrm{CO}_{2}$ at $37^{\circ} \mathrm{C}$. MDA-MB-231/GEM cells were established through continuous exposure of MDA-MB-231 cells to increasing concentrations of gemcitabine (initial concentration, $12 \mathrm{nM}$; increased to $6 \mu \mathrm{M}$ over 6 months; Sigma-Aldrich; Merck KGaA) at $37^{\circ} \mathrm{C}$. After 6 months, gemcitabine resistance was confirmed by comparison of cell viability between MDA-MB-231/GEM and MDA-MB-231 cells in response to $6 \mu \mathrm{M}$ gemcitabine. The immortal breast cell line MCF10A was purchased from ATCC and cultured in DMEM/F12 media with $10 \%$ horse serum, $20 \mathrm{ng} / \mathrm{ml}$ epidermal growth factor, $100 \mathrm{ng} / \mathrm{ml}$ cholera toxin, $0.01 \mathrm{mg} / \mathrm{ml}$ insulin, $500 \mathrm{ng} / \mathrm{ml}$ hydrocortisone (all from HyClone; GE Healthcare Life Sciences) and $1 \%$ penicillin-streptomycin solution in a humidified atmosphere containing $5 \% \mathrm{CO}_{2}$ at $37^{\circ} \mathrm{C}$.

Overexpression and downregulation of $m i R-205-5 p$. miR-205-5p mimic, miR-negative control (NC) mimic, miR-205-5p inhibitor and miR-NC inhibitor were purchased from Shanghai GenePharma Co., Ltd. The sequences were: miR-205-5p mimic, 5'-UCCUUCAUUCCACCGGAGUCU G-3'; miR-NC mimic, 5'-UCGCUUGGUGCAGGUCGG GAA-3'; miR-205-5p inhibitor, 5'-CAGACUCCGGUGGAA UGAAGGA-3'; miR-NC inhibitor, 5'-CAGUACUUUUGU GUAGUACAA-3'. A total of $50 \mathrm{nM}$ miRNA was transfected into MDA-MB-231 and BT549 cells using Lipofectamine ${ }^{\circledR}$ 2000 reagent (Thermo Fisher Scientific, Inc.) according to manufacturer's protocol for $48 \mathrm{~h}$ at $37^{\circ} \mathrm{C}$. Cells were then subjected to the following experiments.

Overexpression of ERp29. The full-length opening reading frame of ERp29 was amplified from MCF10A cells, non-tumorigenic epithelial cell line expressing the normal sequence for ERp29 and ligated into the pcDNA3.1 plasmid (Invitrogen; Thermo Fisher Scientific, Inc.) by the restriction sites of HindIII and XhoI using the following primer sequences: ERp29 forward, 5'-AAGCTTACTATCGCTTAC CTA-3' and reverse, 5'-CTCGAGTGTTGGCACAAGTGC T-3'. For the overexpression of ERp29, $2 \mu \mathrm{g}$ pcDNA3.1-ERp29 was transfected into cells using the Lipofectamine 2000 reagent (Thermo Fisher Scientific, Inc.) according to manufacturer's protocol and incubated for $48 \mathrm{~h}$ at $37^{\circ} \mathrm{C}$. Cells were then subjected to subsequent experiments.

Cell viability and apoptosis assays. Cell viability assays were performed using the Cell Counting Kit-8 (CCK-8; Dojindo Molecular Technologies, Inc.) according to the manufacturer's instruction. A total of $5 \times 10^{3}$ cells/well were seeded into 96-well plates. After treatment of MDA-MB-231, BT549 and MDA-MB-231/GEM cells with different concentrations of gemcitabine $(10,20,40,80$ and $160 \mathrm{nM})$ for $24 \mathrm{~h}$ at $37^{\circ} \mathrm{C}$, cells were transfected with miRNA and/or cDNA using procedures described as follows: MDA-MB-231 cells and BT549 cells were transfected with miR-205-5p mimic or miR-NC mimic; and MDA-MB-231/GEM cells were co-transfected with miR-205-5p mimic or miR-NC mimic and pcDNA3.1-ERp29 or pcDNA3.1 plasmid. Following $48 \mathrm{~h}$ incubation, $10 \mu \mathrm{l} \mathrm{CCK}-8$ solution was added into each well. Cells were incubated for $2 \mathrm{~h}$ at $37^{\circ} \mathrm{C}$ and the absorbance was measured at a wavelength of $450 \mathrm{~nm}$.

Cell apoptosis assays were performed using the Annexin-V/Dead Cell Apoptosis kit (Invitrogen; Thermo Fisher Scientific, Inc.) according to the manufacturer's protocol. After treatment with gemcitabine $(40 \mathrm{nM})$ or corresponding concentrations of dimethylsulfoxide (DMSO) followed by co-transfection with miR-205-5p mimic or miR-NC mimic and pcDNA3.1-ERp29 or pcDNA3.1 plasmid for $48 \mathrm{~h}$, MDA-MB-231/GEM cells were harvested and washed in cold PBS. Cells were then diluted in $1 \mathrm{X}$ Annexin-binding buffer to $1 \times 10^{6}$ cells $/ \mathrm{ml} ; 100-\mu 1$ samples per assay were prepared. Alexa Fluor 488 annexin $\mathrm{V}(5 \mu \mathrm{l})$ and propidium iodide working solution $(1 \mu \mathrm{l} ; 100 \mu \mathrm{g} / \mathrm{ml})$ were added to the $100-\mu \mathrm{l}$ cell suspensions. Cells were incubated at room temperature for $15 \mathrm{~min}$ and annexin-binding buffer (400 $\mu \mathrm{l})$ was added. Subsequently, stained cells were analyzed using a BD FACSCalibur flow cytometer (BD Biosciences). The results were quantified using Cell Quest software (version 5.1; BD Biosciences).

$R N A$ extraction and reverse transcription-quantitative polymerase chain reaction ( $R T-q P C R)$ for miRNA and $m R N A$ assays. Total RNA was extracted from cultured cells using TRIzol ${ }^{\circledR}$ reagent (Invitrogen; Thermo Fisher Scientific, Inc.) and cDNA was synthetized using PrimeScript ${ }^{\mathrm{TM}}$ RT reagent kit (Takara Bio, Inc.) according to the manufacturer's protocol. RT-qPCR reactions were performed in triplicate using SYBR ${ }^{\circledR}$ Premix Ex Taq (Takara Bio, Inc.) in a Bio-Rad CFX96 Real-Time PCR system (Bio-Rad Laboratories, Inc.). The thermocycling conditions used for qPCR were described as follows: Initial denaturation at $95^{\circ} \mathrm{C}$ for $30 \mathrm{sec}$, followed by 40 cycles of $95^{\circ} \mathrm{C}$ for $5 \mathrm{sec}$ and $60^{\circ} \mathrm{C}$ for $30 \mathrm{sec}$. Levels of miR-205-5p and ERp29 were normalized to GAPDH and U6, respectively. The $2^{-\Delta \Delta \mathrm{Cq}}$ method was used to quantify gene 
expression levels (23). The primer sequences used in the present study were listed as follows: miR-205-5p forward, 5'-TCCTTCATTCCACCGGAGTCTG-3' and reverse, 5'-GCG AGCACAGAATTAATACGAC-3'; U6 forward, 5'-ATTGGA ACGATACAGAGAAGATT-3' and reverse, 5'-GGAACGCTT CACGAATTTG-3'; ERp29 forward, 5'-AAAGCAAGTTCG TCTTGGTGA-3' and reverse, 5'-CGCCATAGTCTGAGATCC CCA-3'; GAPDH forward, 5'-TTGGTATCGTGGAAGGAC TCA-3' and reverse, 5'-TGTCATCATATTTGGCAGGTT-3'.

Western blot analysis. ERp29 antibody (cat. no. 37555; 1:1,000) and HSP27 (cat. no., 41043; 1:1,000) were purchased from Signalway Antibody LLC, and GAPDH mouse monoclonal antibody (cat. no. ab8245; 1:10,000) was obtained from Abcam. Anti-mouse (cat. no. CW0221S; 1:10,000) and anti-rabbit (cat.no.CW0234S; 1:10,000) secondary antibodies were provided by CWBiotech. Western blotting was performed as follows: Harvested cells were washed twice with cold PBS and incubated with a cold RIPA buffer (Beyotime Institute of Biotechnology) containing protease inhibitor cocktail (Sigma-Aldrich; Merck $\mathrm{KGaA}$ ) for lysis on ice for $30 \mathrm{~min}$. Subsequently, lysates were centrifuged at $12,000 \mathrm{xg}$ for $15 \mathrm{~min}$ at $4^{\circ} \mathrm{C}$. A bicinchoninic acid protein assay kit (Thermo Fisher Scientific, Inc.) was used to determine the protein concentration in the supernatant. Equal amounts of protein $(20 \mu \mathrm{g} /$ well $)$ were separated by SDS-PAGE (8\% gel), transferred to polyvinylidene fluoride membranes (EMD Millipore; Merck KGaA) and incubated with the aforementioned primary antibodies at $4^{\circ} \mathrm{C}$ overnight and corresponding secondary antibodies at room temperature for $2 \mathrm{~h}$. Blots were developed using SuperSignal West Femto Maximum Sensitivity substrate (Thermo Fisher Scientific, Inc.) and images were recorded using ImageQuant LAS 4000 (GE Healthcare Life Sciences). Densitometry was quantified with ImageJ software (version. 1.8.0; National Institutes of Health).

Dual-luciferase reporter gene assays. Using the TargetScan (release 7.1, www.targetscan.org) miRNA target prediction database, a putative binding site of miR-205-5p was predicted within the 3'-UTR of ERp29. The ERp29 3'-UTR region was amplified using cDNA from MCF10A cells and cloned into the pGL3 vector [wild-type (WT)-ERp29-3'-UTR; Promega Corporation]. Two site mutations were introduced to WT-ERp29-3'-UTR to construct the mutant (Mut) ERp29-3'-UTR. MDA-MB-231 cells were co-transfected with $100 \mathrm{nM}$ of WT-ERp29-3'-UTR or Mut-ERp29-3'-UTR plasmid and $100 \mathrm{nM}$ of miR-205-5p mimic or negative control using Lipofectamine 2000 reagent. Luciferase activity was evaluated $48 \mathrm{~h}$ following transfection using the Dual-Glo Luciferase assay system (Promega Corporation), with all luciferase activity normalized to that of Renilla luciferase activity.

Statistical analysis. All experiments were performed in triplicate. Data were analyzed using GraphPad Prism 5.0 (GraphPad Software, Inc.) and are presented as the mean \pm standard deviation. Paired Student's t-test was used to analyze paired samples from patients with breast cancer. Unpaired Student's t-test was used to analyze differences between two independent groups. Pearson's correlation analysis was used to analyze the correlation between miR-205-5p and ERp29 mRNA expression levels in tumor tissues. One-way analysis of variance followed by Newman Keuls test was performed for comparisons among multiple groups. $\mathrm{P}<0.05$ was considered to indicate a statistically significant difference.

\section{Results}

Low miR-205-5p expression is detected in breast cancer tissues and cells. miR-205-5p expression in breast cancer and matched normal tissues collected from 25 patients with breast cancer was compared using RT-qPCR. It was observed that miR-205-5p levels were significantly decreased in tumor compared with non-tumor tissues (Fig. 1A). Subsequently, relative miR-205-5p expression was determined in MDA-MB-231 and BT549 breast cancer cells and in MCF10A immortalized normal breast cells. The results revealed that miR-205-5p expression levels in MDA-MB-231 and BT549 were lower compared with MCF10A cells (Fig. 1B). Collectively, these finding suggested that miR-205-5p expression decreased in breast cancer tissues and cells compared with normal controls.

miR-205-5p overexpression increases gemcitabine sensitivity of breast cancer cells. To investigate the role of miR-205-5p in breast cancer gemcitabine resistance, a miR-205-5p mimic was transfected into MDA-MB-231 cells and RT-qPCR was performed to detect miR-205-5p mRNA levels. The results demonstrated that the miR-205-5p mimic increased miR-205-5p expression in MDA-MB-231 and BT549 cells compared with the miR-NC mimic group (Fig. 2A). Overexpression of miR-205-5p enhanced gemcitabine-induced cell viability reduction in MDA-MB-231 and BT549 cells (Fig. 2B and C). These data indicated that miR-205-5p overexpression may increase gemcitabine sensitivity of breast cancer cells.

miR-205-5p downregulates ERp29 in breast cancer cells. The current study explored the association between miR-205-5p and ERp29 in breast cancer. RT-qPCR was used to confirm that miR-205-5p inhibitor significantly downregulated miR-205-5p expression in MDA-MB-231 cells compared with the miR-NC inhibitor group (Fig. 3A). Inhibition of miR-205-5p markedly enhanced ERp29 mRNA (Fig. 3B) and protein (Fig. 3C and D) levels. miR-205-5p overexpression decreased ERp29 mRNA (Fig. 3E) and protein (Fig. 3F and G) levels. Taken together, these data indicated that miR-205-5p negatively regulated ERp29 expression levels.

miR-205-5p regulates ERp29 by binding to the 3'-UTR. According to TargetScan, ERp29 is a potential target gene of miR-205-5p. A putative binding site for miR-205-5p was predicted within the 3'-UTR of ERp29 (Fig. 4A). ERp29 WT and Mut 3'-UTR luciferase reporter gene plasmids were constructed to verify this potential binding. Dual-luciferase reporter gene assay results revealed that the miR-205-5p mimic significantly decreased the luciferase activity in the WT-ERp29-3'-UTR co-transfection system in MDA-MB-231 cells compared with the miR-NC mimic transfection (Fig. 4B).

miR-205-5p expression is decreased in MDA-MB-231/GEM cells. MDA-MB-231/GEM cells were established to study the mechanisms of gemcitabine resistance. With increasing gemcitabine concentration, MDA-MB-231/GEM viability was 

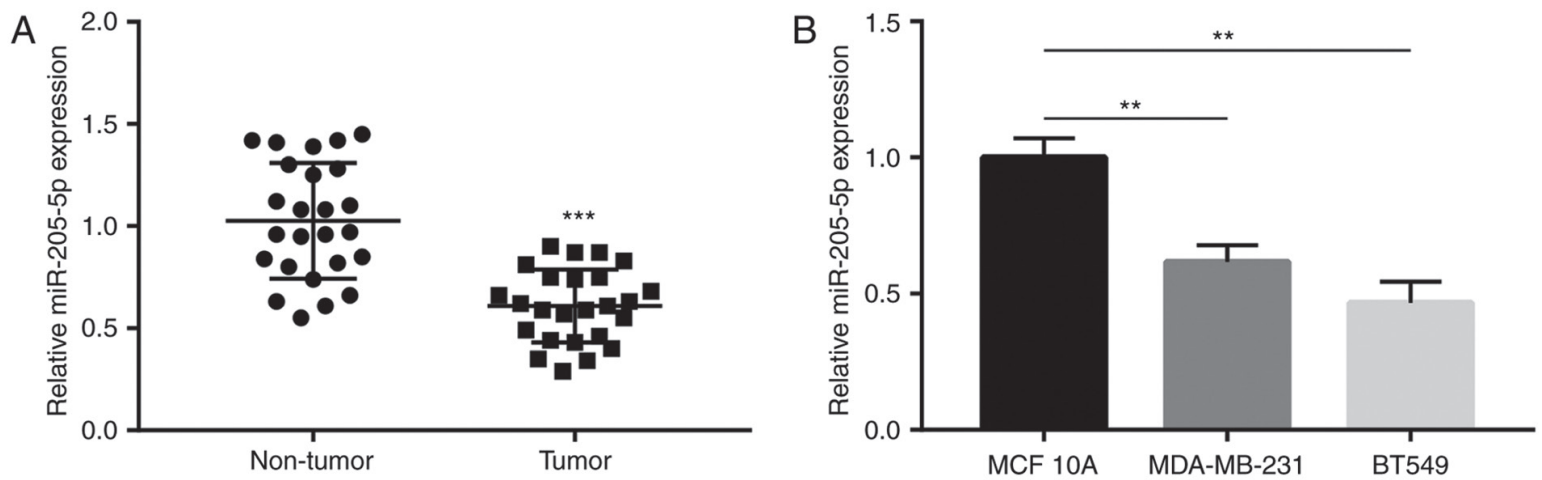

Figure 1. miR-205-5p is downregulated in breast cancer tissues and cell lines. (A) miR-205-5p expression was decreased in tumor tissues compared with matched normal tissues collected from 25 patients with breast cancer. (B) miR-205-5p expression was decreased in MDA-MB-231 and BT549 cell lines compared with the MCF10A cell line. ${ }^{* *} \mathrm{P}<0.01$ and ${ }^{* * *} \mathrm{P}<0.001$.
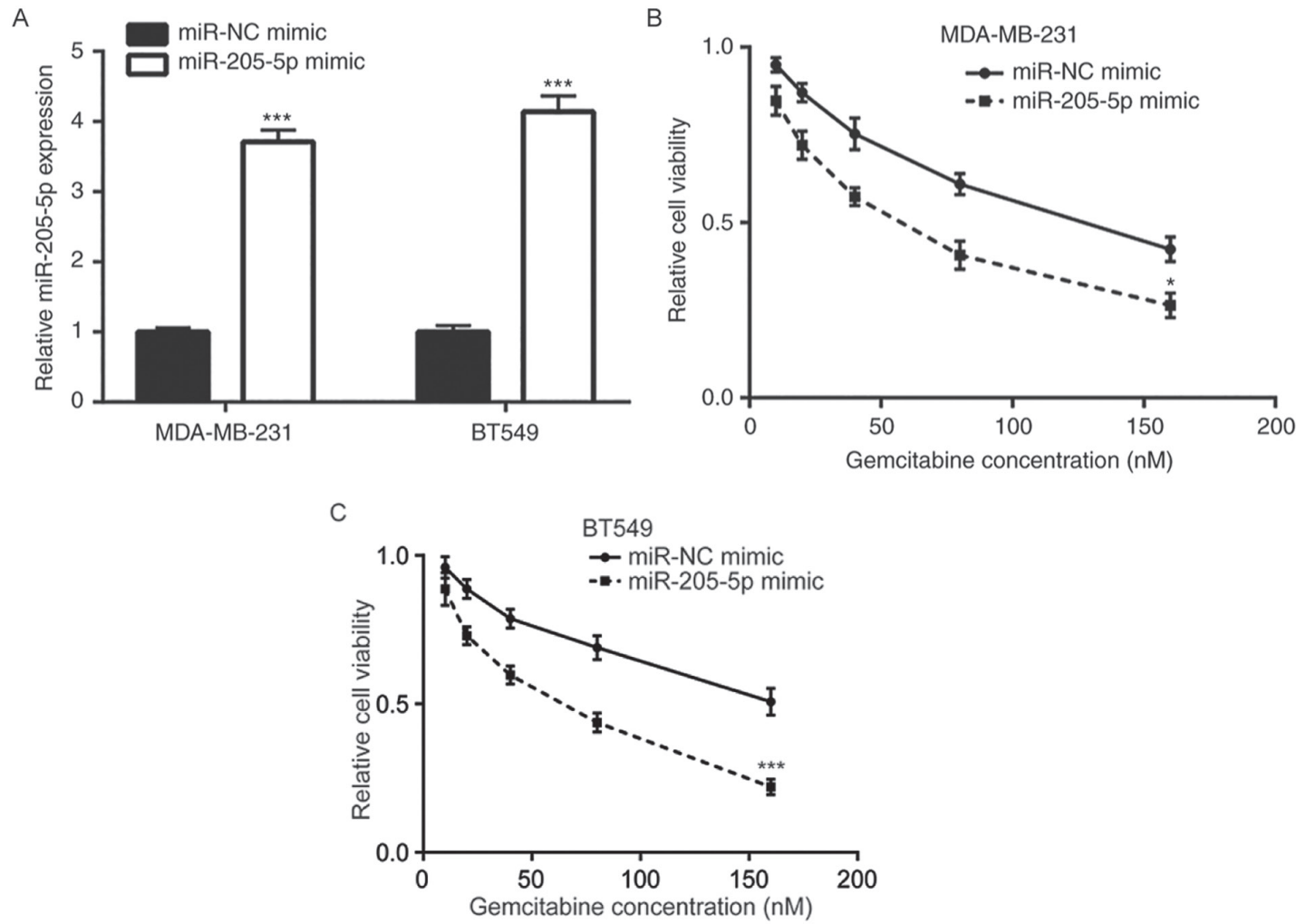

Figure 2. Overexpression of miR-205-5p increases gemcitabine sensitivity of breast cancer cells. (A) Compared with miR-NC mimics, miR-205-5p mimics significantly increased miR-205-5p expression in MDA-MB-231 and BT549 cells. miR-205-5p mimics enhanced the gemcitabine-induced cell viability reduction in (B) MDA-MB-231 and (C) BT549 cells. "P<0.05 and ${ }^{* * * *} \mathrm{P}<0.001$ vs. the miR-NC mimic group. NC, negative control; miR, microRNA.

significantly greater compared with MDA-MB-231/P cells, suggesting MDA-MB-231/GEM cells were comparatively less insensitive towards gemcitabine treatment (Fig. 5A). ERp29 mRNA and protein expression levels were notably increased in MDA-MB-231/GEM cells compared with the MDA-MB-231/P group (Fig. 5B-D). HSP27 is regulated by ERp29 and involved in breast cancer drug resistance $(12,24)$. In the current study, it was observed that HSP27 protein levels significantly increased in MDA-MB-231/GEM cells compared with MDA-MB-231/P cells (Fig. 5C and D). In addition, miR-205-5p expression significantly decreased in MDA-MB-231/GEM cells compared with MDA-MB-231/P cells (Fig. 5E). miR-205-5p downregulation and ERp29 upregulation in MDA-MB-231/GEM cells suggested that miR-205-5p/ERp29 may be involved in the development of gemcitabine resistance in breast cancer cells.

miR-205-5p overexpression reverses gemcitabine resistance of MDA-MB-231/GEM cells via ERp29 repression. pcDNA3.1-ERp29 recombinant plasmids were constructed and co-transfected with the miR-205-5p mimic into MDA-MB-231/GEM cells to confirm the aforementioned results. The results demonstrated that miR-205-5p overexpression reduced ERp29 protein expression levels; a phenomenon that was reversed by co-transfection with pcDNA3.1-ERp29 recombinant plasmid (Fig. 6A and B). To evaluate the function of miR-205-5p in the development of gemcitabine 

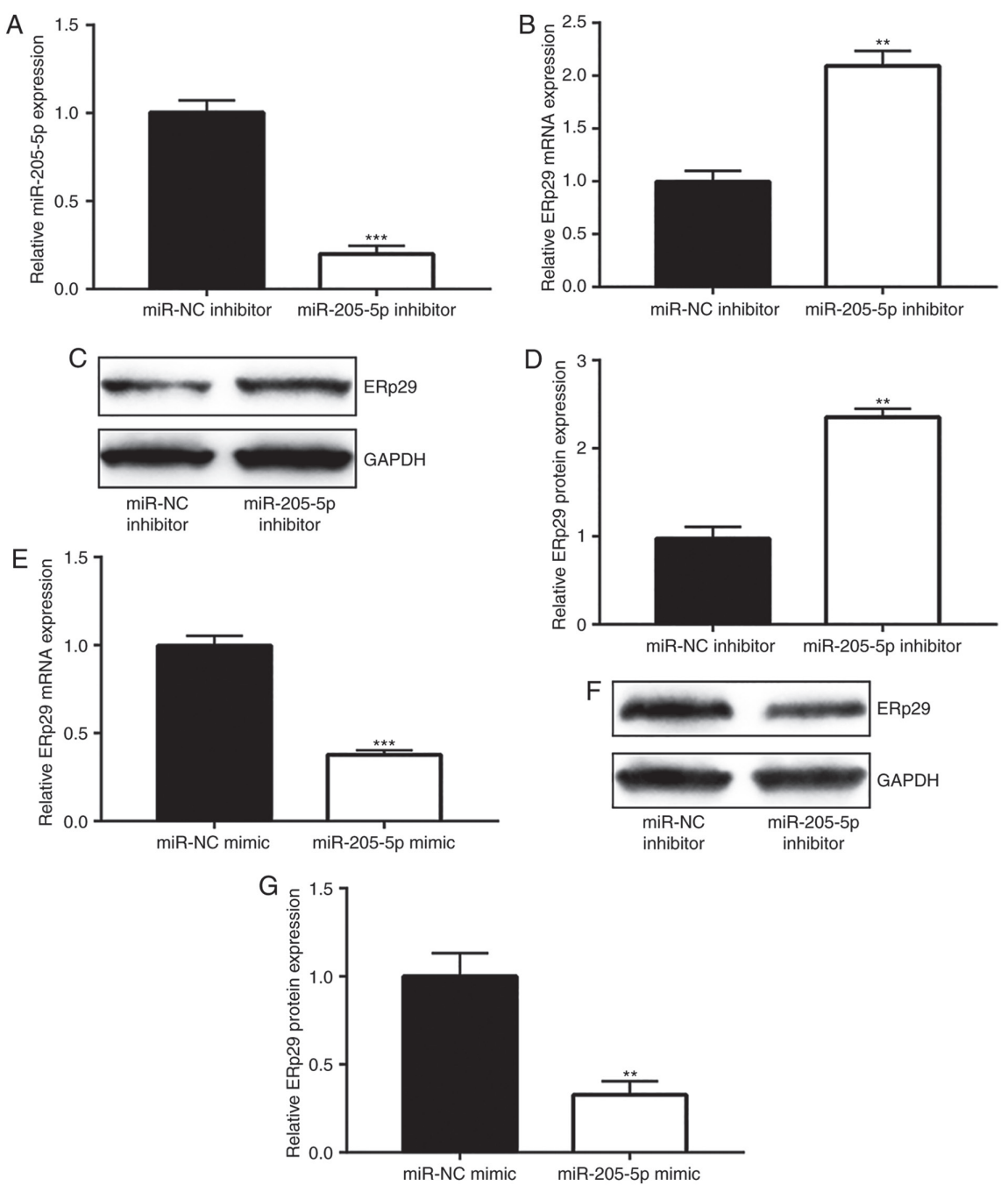

Figure 3. miR-205-5p negatively regulates ERp29 in breast cancer cells. (A) miR-205-5p inhibitor significantly inhibited miR-205-5p expression in the MDA-MB-231 cell line. (B) RT-qPCR results indicated that miR-205-5p inhibitor enhanced ERp29 mRNA expression levels in the MDA-MB-231 cell line. Protein expression levels of ERp29 were determined using (C) western blotting and (D) quantified. (E) RT-qPCR results indicated that miR-205-5p mimic decreased ERp29 mRNA expression levels. Protein expression levels of ERp29 in the MDA-MB-231 cell line were determined using $(F)$ western blotting and (G) analyzed quantitatively. ${ }^{* *} \mathrm{P}<0.01$ and ${ }^{* * *} \mathrm{P}<0.001$ vs. the respective control group. RT-qPCR, reverse transcription-quantitative PCR; NC, negative control; miR, microRNA; ERp29, endoplasmic reticulum protein 29.

\section{A ERp29-3'-UTR 5'-AUAACCUCUCAAGUGUGAAGGAG-3' 促}

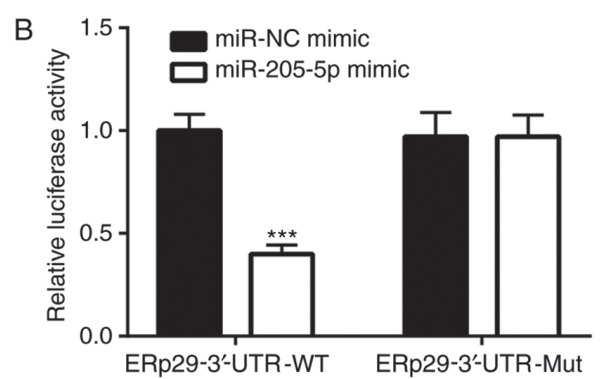

Figure 4. miR-205-5p regulates ERp29 by binding to its 3'-UTR. (A) Bioinformatic prediction of the putative binding site of miR-205-5p on the 3'-UTR of ERp29. (B) Dual-luciferase assay confirmed that the luciferase activity was reduced in MDA-MB-231 cells co-transfected with miR-205-5p mimic and WT-ERp29-3'-UTR reporter plasmid and was unaltered in cells co-transfected with miR-205-5p mimic and Mut-ERp29-3'-UTR reporter plasmid. ${ }^{* * *} \mathrm{P}<0.001$ vs. the miR-NC mimic. 3'-UTR, 3'untranslated region; WT, wild-type; Mut, mutant; NC, negative control; miR, microRNA; ERp29, endoplasmic reticulum protein 29. resistance, cell apoptosis and viability assays were performed to detect effects of miR-205-5p and ERp29 overexpression on gemcitabine sensitivity in breast cancer cells. The results revealed that among cells treated with gemcitabine, miR-205-5p overexpression significantly enhanced gemcitabine-induced apoptosis in MDA-MB-231/GEM cells, which was reversed following transfection with recombinant ERp29. Among cells treated with DMSO, there were no significant differences between the 3 different groups. In addition, gemcitabine significantly increased apoptosis in cells transfected with miR-205-5p mimic + pcDNA3.1 but not in miR-NC mimic + pcDNA3.1 or miR-205-5p mimic + pcDNA3.1-ERp29 groups (Fig. 6C and D). Meanwhile, miR-205-5p overexpression significantly potentiated the inhibitory effects of gemcitabine on cell viability in MDA-MB-231/GEM cells, which was significantly reversed by transfection with recombinant ERp29 (Fig. 6E). These findings indicate that miR-205-5p overexpression sensitized MDA-MB-231/GEM cells to gemcitabine by suppressing ERp29 expression. 

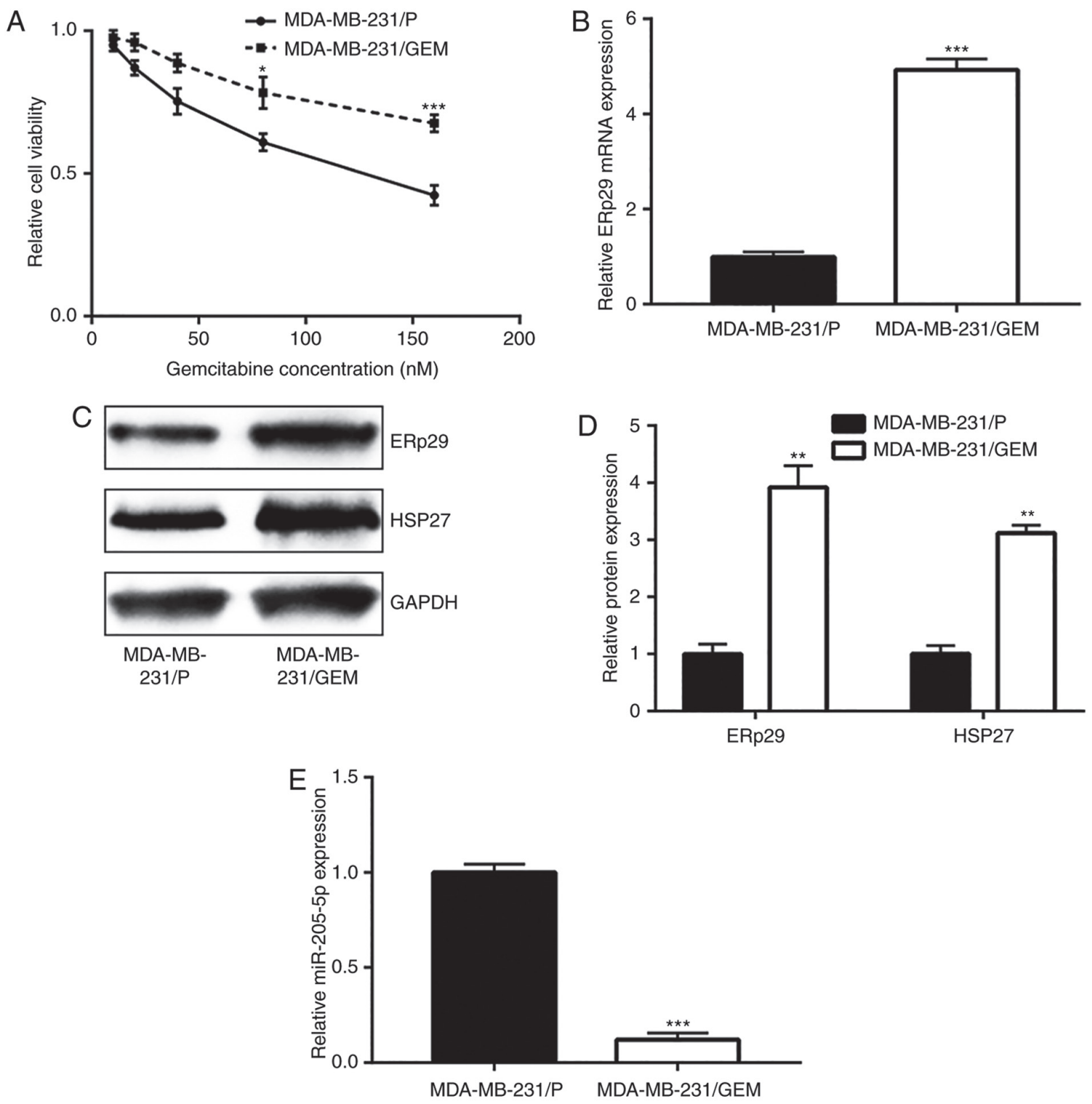

Figure 5. Downregulation of miR-205-5p in MDA-MB-231/GEM cells. (A) Compared with MDA-MB-231/P cells, the cell viability of MDA-MB-231/GEM cells was relatively higher. (B) mRNA expression of ERp29 increased in the MDA-MB-231/GEM cell line compared with the DMA-MB-231/P cell line. Protein expression of ERp29 and HSP27 increased in the MDA-MB-231/GEM cell line compared with the DMA-MB-231/P cell line as demonstrated by (C) western blotting and (D) quantitative analysis. (E) miR-205-5p expression significantly decreased in the MDA-MB-231/GEM cell line compared with the MDA-MB-231/P cell line. "P<0.05, ${ }^{* *} \mathrm{P}<0.01$ and ${ }^{* * * *} \mathrm{P}<0.001$ vs. the MDA-MB-231/P group. MDA-MB-231/GEM, gemcitabine-resistant breast cancer cell line; MDA-MB-231/P parental breast cancer cell line; miR, microRNA; ERp29, endoplasmic reticulum protein 29; HSP27, heat shock protein 27.

ERp29 expression is elevated in breast cancer tissues and negatively correlated with miR-205-5p expression. To further study the association between miR-205-5p and ERp29 in breast cancer tissues, RT-qPCR was used to detect ERp29 mRNA levels in 25 paired tumor and normal tissues. It was observed that ERp29 expression was significantly elevated in tumor tissues compared with non-tumor samples (Fig. 7A). Furthermore, ERp29 mRNA expression was negatively correlated with the expression of miR-205-5p, as determined by analyzing the expression data of 25 breast cancer tissues (Fig. 7B).

\section{Discussion}

Breast cancer is one of the leading causes of cancer-associated mortality among women worldwide $(1,2)$. Despite advances in breast cancer chemotherapy (7), the development of chemotherapy resistance remains a major obstacle in successful breast cancer treatment (8). Gemcitabine is a widely used chemotherapy drug that serves an important role in the treatment of advanced breast cancer (6). However, its therapeutic use in cancer chemotherapy is impeded, at least in part, by drug resistance (25). Over the past decade, increasing research efforts focused on exploring the underlying mechanisms of drug resistance. Recently, various studies have suggested that miRNAs serve an important role in chemotherapy resistance $(26,27)$. miRNAs are tumor suppressors or oncogenes and are able to modulate cancer progression, including cell proliferation and apoptosis $(16,28)$. Certain miRNAs, including let-7, miR-122, miR-152 and miR-1246, have been reported to modulate liver cancer stem cells by directly or indirectly binding to specific target genes that are involved in signal pathways, including the 

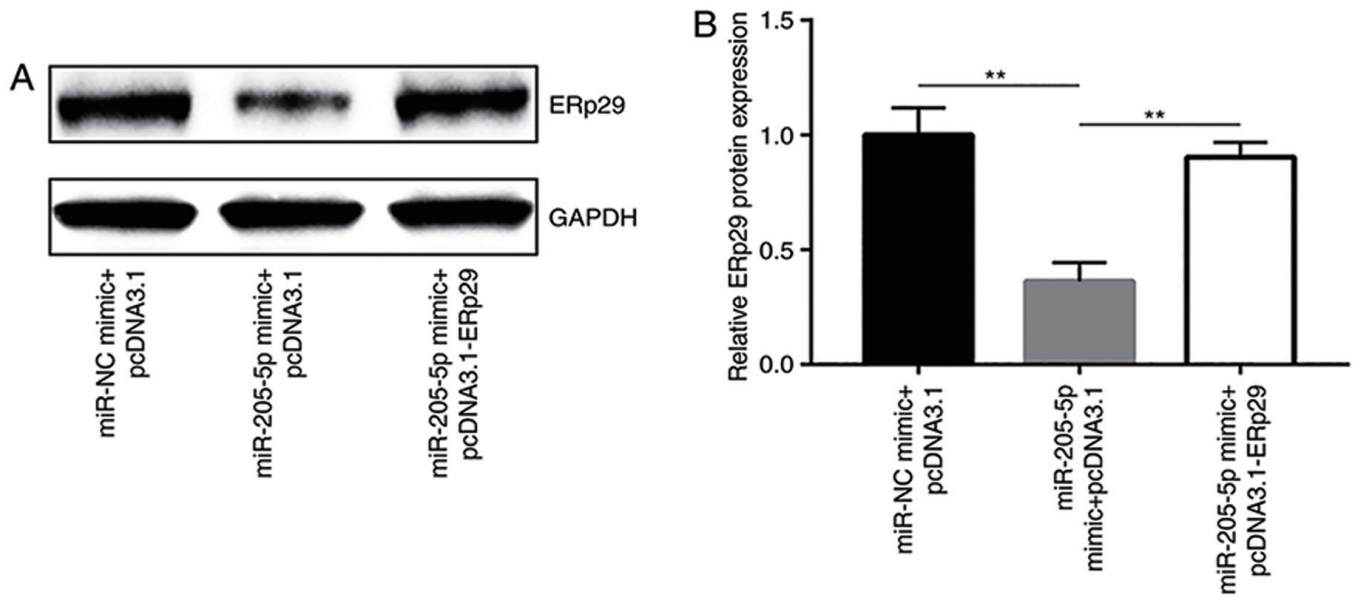

C

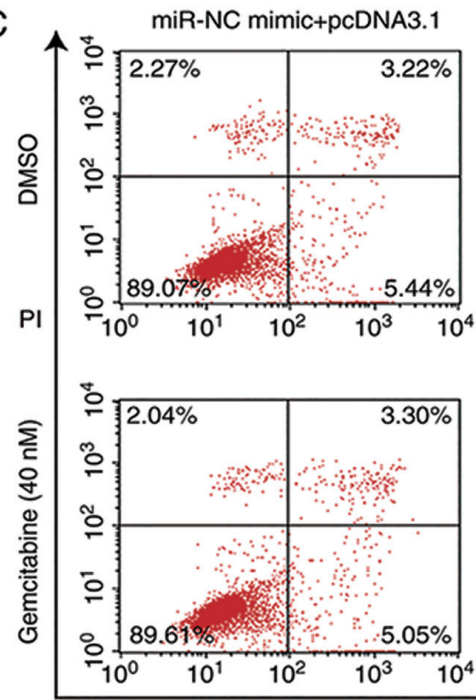

miR-205-5p mimic+pcDNA3.1
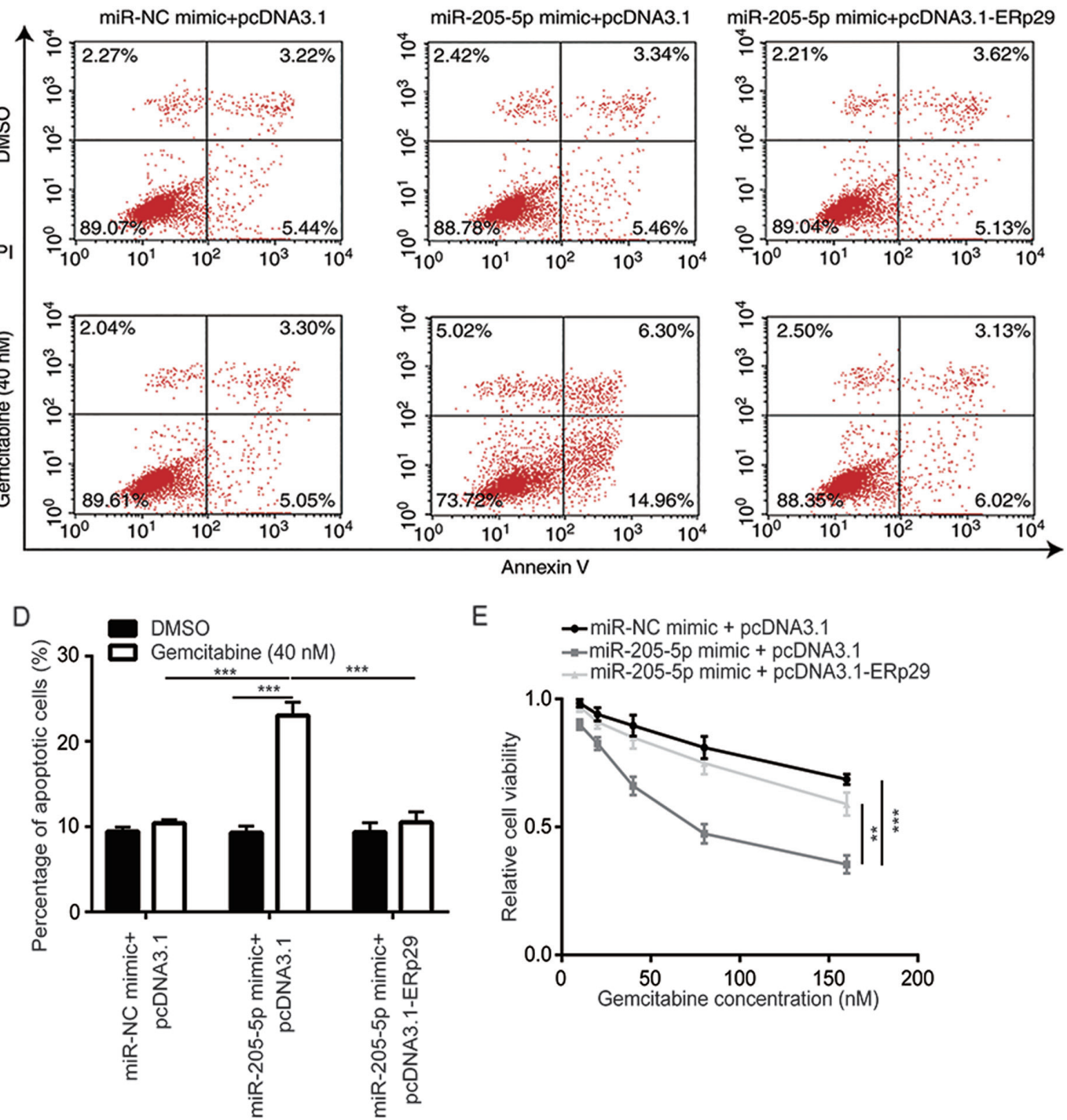

$E$

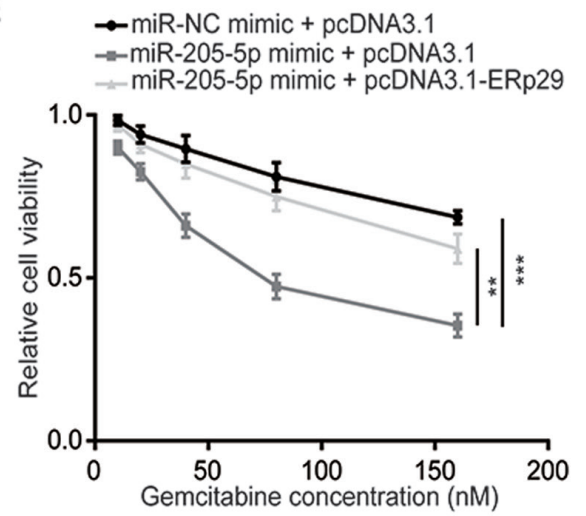

Figure 6. Overexpression of miR-205-5p reverses gemcitabine resistance of MDA-MB-231/GEM cells via repression of ERp29. Compared with the miR-NC mimic + pcDNA3.1 group, protein expression level of ERp29 decreased in the miR-205-5p mimic + pcDNA3.1 group and this effect was reversed by transfection with miR-205-5p mimic + pcDNA3.1-ERp29 as demonstrated by (A) western blotting and (B) quantitative analysis. Cell apoptosis of the transfected cells was analyzed by (C) flow cytometry and (D) quantified. (E) Cell viability was measured using Cell Counting Kit-8. ${ }^{* *} \mathrm{P}<0.01$ and ${ }^{* * * *} \mathrm{P}<0.001$. NC, negative control; miR, microRNA; ERp29, endoplasmic reticulum protein 29; PI, propidium iodide.

Wnt $/ \beta$-catenin signaling, TGF- $\beta$ signaling, JAK/STAT signaling and epithelial-mesenchymal transition (EMT) pathway (27). Yu et al (29) reported that miR-200a was overexpressed in gemcitabine-resistant breast cancer cells and that miR-200a inhibition restored sensitivity. In this study, miR-200a promoted DNA damage resistance by inhibiting DNA damage-induced 

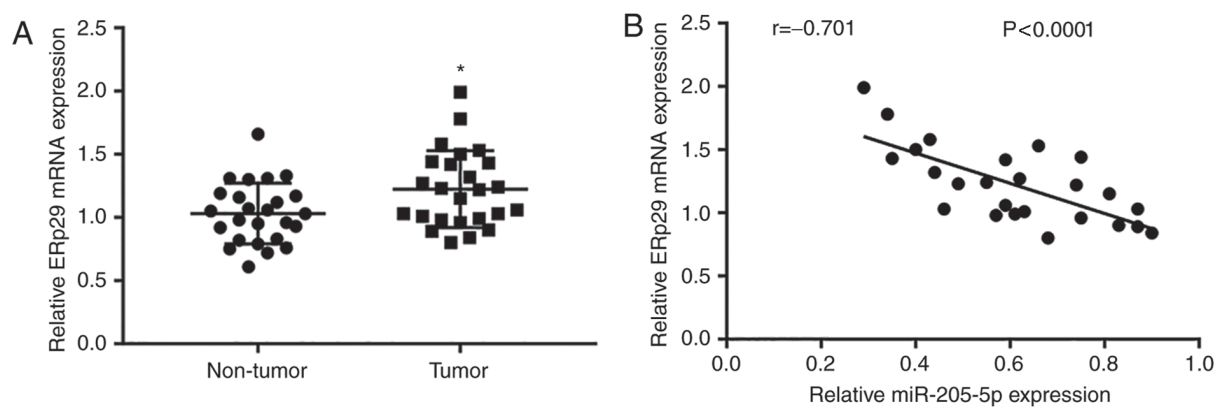

Figure 7. ERp29 mRNA levels are elevated in breast cancer tissues and negatively correlated with miR-205-5p levels. (A) ERp29 mRNA levels were elevated in tumor tissues compared with matched adjacent normal tissues from 25 patients with breast cancer. (B) ERp29 mRNA levels were negatively correlated with miR-205-5p levels in tumor tissues from 25 patients with breast cancer. ${ }^{~} \mathrm{P}<0.05$. miR, microRNA; ERp29, endoplasmic reticulum protein 29.

apoptosis via Yes associated protein 1 and tumor protein p53 inducible nuclear protein 1 . Previous studies revealed that miR-205-5p is one of the most researched miRNAs in breast cancer and it regulates cell proliferation and invasion $(21,30)$. $\mathrm{Li}$ and $\mathrm{Li}$ (31) reported that miR-205-5p inhibited cell migration and invasion in prostate carcinoma by targeting zinc finger E-box binding homeobox (ZEB) 1. Previous studies further reported that compared with differentiated tumor cells, patient-derived breast cancer stem cells expressed higher levels of miR-205-5p (22). miR-205-5p upregulation controls cancer stem cell phenotype by targeting erb-b2 receptor tyrosine kinase 2, tumor protein p63 and epidermal growth factor receptor contributing to targeted therapy resistance (24). miR-205 accumulation confers gemcitabine sensitivity and in gemcitabine-resistant cancer stem cells it targets and suppresses ZEB1/2, E2F transcriptional factor 1, erb-b2 receptor tyrosine kinase 3 and vascular endothelial growth factor A (32). However, the effects of miR-205-5p on gemcitabine resistance in breast cancer cells remain to be elucidated. In the current study, using cell viability assays, miR-205-5p overexpression in combination with gemcitabine treatment at varying concentration was used to investigate gemcitabine-sensitivity of breast cancer cells. miR-205-5p overexpression increased gemcitabine sensitivity in breast cancer cells. Additionally, miR-205-5p was downregulated in the established MDA-MB-231/GEM cells and induced miR-205-5p overexpression reversed gemcitabine resistance of MDA-MB-231/GEM cells. Furthermore, in the present study, miR-205-5p overexpression increased apoptosis in MDA-MB-231/GEM cells following treatment with $40 \mathrm{nM}$ gemcitabine. The results revealed the role of miR-205-5p in breast cancer gemcitabine resistance.

The current study reported that ERp29 was predicted as target gene of miR-205-5p according to the TargetScan database. Transfection with WT and Mut ERp29-3'-UTR luciferase reporter gene plasmids verified that miR-205-5p regulated ERp29 by binding to the 3'-UTR. ERp29 is negatively correlated with breast cancer development $(33,34)$ and functions as a tumor suppressor $(35,36)$. ERp29 overexpression aids tumor cell survival by inhibiting genotoxic effects caused by chemotherapy (37). Zhang and Putti (12) investigated the effects of breast cancer cell chemotherapeutic agents on ERp29 expression. ERp29 expression increased doxorubicin resistance and decreased doxorubicin-induced apoptosis in MDA-MB-231 cells, and ERp29 knockdown in MCF-7 cells increased the cytotoxicity of doxorubicin. In the current study, overexpression and inhibition of miR-205-5p suggested that ERp29 mRNA and protein levels were negatively regulated by miR-205-5p in breast cancer cells. In addition, miR-205-5p overexpression-induced apoptosis and decreased cell viability were reversed by increased ERp29 expression.

In conclusion, it was revealed that miR-205-5p decreased breast cancer gemcitabine sensitivity in vitro via regulation of ERp29, suggesting that miR-205-5p may serve as a potential treatment target in breast cancer.

\section{Acknowledgments}

Not applicable.

\section{Funding}

No funding was received.

\section{Availability of data}

The datasets used and/or analyzed during the current study are available from the corresponding author on reasonable request.

\section{Author's contribution}

$\mathrm{CM}$ and GW contributed to study design and supervision. CM, XS and WG were responsible for acquisition of data. GW performed interpretation of data. Clinical sample collection and analysis was performed by WG and FF. CM and GW prepared the manuscript. All authors read and approved the final manuscript.

\section{Ethics approval and consent to participate}

The present study was approved by the Ethics Committee of Tianjin Baodi People's Hospital. All patients provided written consent before being enrolled into the study.

\section{Patient consent for publication}

Written informed consent for publication was provided by all participants.

\section{Competing interests}

The authors declare that they have no competing interests. 


\section{References}

1. Smith IE, Dowsett M, Ebbs SR, Dixon JM, Skene A, Blohmer JU, Ashley SE, Francis S, Boeddinghaus I and Walsh G; IMPACT Trialists Group: Neoadjuvant treatment of postmenopausal breast cancer with anastrozole, tamoxifen, or both in combination: The immediate preoperative anastrozole, tamoxifen, or combined with tamoxifen (IMPACT) multicenter double-blind randomized trial. J Clin Oncol 23: 5108-5116, 2005.

2. Gralow JR, Burstein HJ, Wood W, Hortobagyi GN, Gianni L, von Minckwitz G, Buzdar AU, Smith IE, Symmans WF, Singh B and Winer EP: Preoperative therapy in invasive breast cancer: Pathologic assessment and systemic therapy issues in operable disease. J Clin Oncol 26: 814-819, 2008.

3. Tripathy D: Gemcitabine as single-agent therapy for advanced breast cancer. Clin Breast Cancer 1 (Suppl): S8-S11, 2002.

4. Spielmann M: Single-agent gemcitabine as first line therpy in patient with metastatic breast cancer. Oncology 62 2-8, 2011.

5. Blackstein M, Vogel CL, Ambinder R, Cowan J, Iglesias J and Melemed A: Gemcitabin as first-line therapy in patient with metastatic breast cancer: A phase II trail. Oncology 62: 2-8, 2002.

6. CACA-CBCS, Chinese Anti-Cancer Association, Committee of Breast Cancer Society: Guidelines for diagnosis and treatment of breast cancer in China (2017). China Oncol 27: 1007-3639, 2017.

7. Vincenzi B, Santini D, Spoto S, Finolezzi E, D'Angelillo RM, La Cesa A and Tonini G: The antineoplastic treatment in the elderly. Clin Ter 153: 207-215, 2002.

8. Wu ZH, Lin C, Liu MM, Zhang J, Tao Z and Hu XC: Src inhibition can synergize with gemcitabine and reverse resistance in triple negative breast cancer cells via the AKT/c-Jun pathway. PLoS One 11: e0169230, 2016.

9. Zhang D and Richardson DR: Endoplasmic reticulum protein 29 (ERp29): An emerging role in cancer. Int J Biochem Cell Biol 43: 33-36, 2011.

10. Martin C, Ardizzoni A and Rosso R: Gemcitabine: Safety profile and efficacy in non-small cell lung cancer unaffected by age. Aging (Milano) 9: 297-303, 1997.

11. Wu P, Zhang H, Qi L, Tang Q, Tang Y, Xie Z, Lv Y, Zhao S and Jiang W: Identification of ERp29 as a biomarker for predicting nasopharyngeal carcinoma response to radiotherapy. Oncol Rep 27: 987-994, 2012.

12. Zhang D and Putti TC: Over-expression of ERp29 attenuates doxorubicin-induced cell apoptosis through up-regulation of Hsp27 in breast cancer cells. Exp Cell Res Dec 316: 3522-3531, 2010.

13. Mori-Iwamoto S, Kuramitsu Y, Ryozawa S, Mikuria K, Fujimoto M, Maehara S, Maehara Y, Okita K, Nakamura K and Sakaida I: Proteomics finding heat shock protein 27 as a biomarker for resistance of pancreatic cancer cells to gemcitabine. Int J Oncol 31: 1345-1350, 2007.

14. Zhang Y, Hu Y, Wang JL, Yao H, Wang H, Liang L, Li C, Shi H, Chen Y, Fang JY and Xu J: Proteomic identification of ERP29 as a key chemoresistant factor activated by the aggregating p53 mutant Arg282Trp. Oncogene 36: 5473-5483, 2017.

15. Ambros V: The functions of animal microRNAs. Nature 431: 350-355, 2004

16. Hammond SM: An overview of microRNAs. Adv Drug Deliv Rev 87: 3-14, 2015.

17. Calin GA and Croce CM: MicroRNA signatures in human cancers. Nat Rev Cancer 6: 857-866, 2006.

18. Calin GA and Konopleva M: Small gene, big number, many effects. Blood 120: 240-241, 2012.

19. Farmer DT, Shariat N, Park CY, Liu HJ, Mavropoulos A and McManus MT: Partially penetrant postnatal lethality of an epithelial specific MicroRNA in a mouse knockout. PLoS One 8 e76634, 2013.
20. Greene SB, Gunaratne PH, Hammond SM and Rosen JM: A putative role for microRNA-205 in mammary epithelial cell progenitors. J Cell Sci 123: 606-618, 2010.

21. Wu H, Zhu S and Mo YY: Suppression of cell growth and invasion by miR-205 in breast cancer. Cell Res 19: 439-448, 2009.

22. De Cola A, Volpe S, Budani MC, Ferracin M, Lattanzio R, Turdo A, D'Agostino D, Capone E, Stassi G, Todaro M, et al: MIR-205-5p-mediated downregulation of ErbB/HER receptors in breast cancer stem cells results in targeted therapy resistance. Cell Death 6: e1823, 2015 .

23. Livak KJ and Schmittgen TD: Analysis of relative gene expression data using real-time quantitative PCR and the 2(-Delta Delta C(T)) method. Methods 25: 402-408, 2001

24. Qi L, Wu P, Zhang X, Qiu Y, Jiang W, Huang D, Liu Y, Tan P and Tian Y: Inhibiting ERp29 expression enhances radiosensitivity in human nasopharyngeal carcinoma cell lines. Med Oncol 29: 721-728, 2012.

25. Dyawanapelly S, Kumar A and Chourasia MK: Lessons learned from gemcitabine: Impact of therapeutic carrier systems and Gemcitabine's drug conjugates on cancer therapy. Crit Rev Ther Drug Carrier Syst 34: 63-96, 2017.

26. Ying SY, Chang DC and Lin SL: The microRNA (miRNA): Overview of the RNA genes that modulate gene function. Mol Biotechnol 38: 257-268, 2008.

27. Lou W, Liu J, Gao Y, Zhong G, Ding B, Xu L and Fan W: MicroRNA regulation of liver cancer stem cells. Am J Cancer Res 8: 1126-1141, 2018.

28. Zhang W, Liu J and Wang G: The role of microRNAs in human breast cancer progression. Tumour Biol 35: 6235-6244, 2014.

29. Yu SJ, Yang L, Hong Q, Kuang XY, Di GH and Shao ZM MicroRNA-200a confers chemoresistance by antagonizing TP53INP1 and YAP1 in human breast cancer. BMC Cancer 18: 74, 2018.

30. Gregory PA, Bracken CP, Bert AG and Goodall GJ: MicroRNAs as regulators of epithelial-mesenchymal transition. Cell Cycle 7: 3112-3118, 2008.

31. Li L and Li S: miR-205-5p inhibits cell migration and invasion in prostatic carcinoma by targeting ZEB1. Oncol Lett 16: 1715-1721, 2018.

32. Okamoto K, Miyoshi K and Murawaki Y: miR-29b, miR-205 and miR-221 enhance chemosensitivity to gemcitabine in $\mathrm{HuH} 28$ human cholangiocarcinoma cells. PLoS One 8: e77623, 2013.

33. Bambang IF, Lee YK, Richardson DR and Zhang D: Endoplasmic reticulum protein 29 regulates epithelial cell integrity during the mesenchymal-epithelial transition in breast cancer cells. Oncogene 32: 1240-1251, 2013.

34. Deng YJ, Tang N, Liu C, Zhang JY, An SL, Peng YL, Ma LL, Li GQ, Jiang Q, Hu CT, et al: CLIC4,ERp29, and Smac/DIABLO derived from metastatic cancer stem-like cells stratify prognostic risks of colorectal cancer. Clin Cancer Res 20: 3809-3817, 2014.

35. Bambang IF, Xu S, Zhou J, Salto-Tellez M, Sethi SK and Zhang D: Overexpression of endoplasmic reticulum protein 29 regulates mesenchymal-epithelial transition and suppresses xenograft tumor growth of invasive breast cancer cells. Lab Invest 89: 1229-1242, 2009

36. Shnyder SD, Mangum JE and Hubbard MJ: Triplex profiling of functionally distinct chaperones (ERp29/PDI/BiP) reveals marked heterogeneity of the endoplasmic reticulum proteome in cancer. J Proteome Res 7: 3364-3372, 2008

37. Farmaki E,Mkrtchian S,Papazian I,Papavassiliou AG and Kiaris H: ERp29 regulates response to doxorubicin by a PERK-mediated mechanism. Biochim Biophys Acta 1813: 1165-1171, 2011.

This work is licensed under a Creative Commons Attribution-NonCommercial-NoDerivatives 4.0 International (CC BY-NC-ND 4.0) License. 